\title{
Rapid solar wind and geomagnetic variability during the ascendant phases of the 11-yr solar cycles
}

\author{
G. Maris ${ }^{1}$ and O. Maris ${ }^{2}$ \\ ${ }^{1}$ Institute of Geodynamics, RO-020032, Bucharest, Romania \\ email: gmaris@geodin.ro \\ ${ }^{2}$ Institute for Space Sciences, RO-077125 Bucharest, Romania \\ email: maris@spacescience.ro
}

\begin{abstract}
Solar activity and its consequences for the interplanetary space are governing and perturbing the Earth's magnetosphere. The response of the terrestrial magnetosphere displayed as geomagnetic disturbances is measured by several geomagnetic indices. This paper analyses the geomagnetic variability during the ascendant phases of the last four solar cycles (nos. 20-23) under the influence of the high speed solar wind. The ascendant phase of a solar cycle is a proper interval of the irrespective cycle during which the influences of the solar cycles adjacent to it (the precedent and the following ones) are not present. The correlation between the geomagnetic indices and the high speed stream intensity during the analysed intervals was examined. LombScargle method of spectral analysis was applied on the solar wind velocity series during the ascendant phases.
\end{abstract}

Keywords. Sun: activity, solar wind, solar-terrestrial relations

\section{Introduction}

The solar wind (SW) is governing the heliospheric structure and dynamics; it is "the medium" through which all the solar perturbations travel to Earth and the other solar system planets. It consists of two components, the slow solar wind and the rapid (fast) solar wind. The slow solar wind is characterized by a velocity of about $300-350 \mathrm{~km} / \mathrm{s}$, temperatures of $1.4-1.6 \times 10^{6} \mathrm{~K}$ and a composition similar to the corona. The fast solar wind component presents a typical speed of about $500-750 \mathrm{~km} / \mathrm{s}$, temperatures of $8 \times 10^{5} \mathrm{~K}$ and resembles the composition of the Sun's photosphere. The origin of this highspeed component (High Speed Streams - HSSs) is located in the Coronal Holes (CHs) which tend to appear in regions near to Sun's poles. The HSSs produced by coronal hole are recurrent, co-rotating streams, with an apparent tendency to occur at every 27 days. There also are non-recurrent HSSs produced by some eruptive solar phenomena such as flares, coronal mass ejections and, sudden disappearing filaments. The time variation of the HSS parameters and their occurrence rate shows 11-year periodicity with differences between the two HSS classes, generated by $\mathrm{CH}$ or by eruptive solar phenomena.

The 11-yr solar cycle is the best established cycle from all range of solar periodicities. Some specific indices that evaluate the number and structure of the coronal holes show 11-yr periodicity, which are in antiphase with the Wolf number, for example. It is best seen in the frequency indices' variability. The 11-yr solar cycle can be found in the variability of the geomagnetic indices, too. We emphasize that the phases of 11-yr cycle are not "rigid" components, they could have different durations and intensities in different cycles. A main physical property of the solar magnetic fields is the polar magnetic field reversal every 11-yr cycle that take place after the cycle maximum. This reversal will be 
reflected by the change of the magnetic polarity distribution in the sunspot groups of the two solar hemispheres (North and South) during the next cycle. So, since the reversal epoch, two magnetic dipols with opposite polarities are present on the Sun for a long interval (during descendant and the next minimum phases). The ascendant phase of a solar cycle is the only proper interval of the irrespective cycle during which the influences of the solar cycles adjacent to it (the precedent and the following ones) are not present.

This paper presents a brief analysis of the geomagnetic variability during the ascending phases of the last four solar cycles (nos. 20-23) under the influence of the high speed solar wind. HSS intensity, geomagnetic indices and methods used are presented in Section 2. Results and discussions end paper in Section 3.

\section{Data and Method}

A high-speed stream in the solar wind was defined as the amplification of the solar wind velocity with more than $100 \mathrm{~km} / \mathrm{s}$ lasting for more than 48 hours. Using the HSS parameters given in different catalogues we calculated their importance (I) defined as (Maris \& Maris, 2003): $I=\Delta V \times d$, where $\triangle V=V_{\max }-V_{0}$ and $V_{0}$ - the pre-stream solar wind velocity, $V_{\max }$ - the maximum velocity of the stream (in $\mathrm{km} / \mathrm{s}$ ) and $\mathrm{d}$ - the duration of the streams, in days. We used the catalogues of the HSSs issued for different intervals covering four 11-yr SCs (nos. 20-23, 1964-2007) (Lindblad et al., 1989; Mavromichalaki \& Vassilaki, 1998 and the catalogues cited there; Maris \& Maris, 2008) in order to obtain the monthly values of HSS importance, as sum of the importances of all HSSs registered during given month, $\sum I$. Their 11-yr dynamics is compared with the monthly smoothed sunspot relative numbers (W), obtained from SIDC, Brussels (http://sidc.oma.be/sunspot-data/). The geomagnetic indices Aa (http://www.geomag.bgs.ac.uk/gifs/aaindex.html) and, Ap (http://omniweb.gsfc.nasa.gov/form/dx1.html) were also used.

The HSS impact on the geomagnetic variability is well reflected by cyclic behavior of $\sum I$ and Ap or Aa (Pirvutoiu et al., 2009). Here we look to their relation during the ascendant phases of the SCs 20-23. In this end, we demarcated the 11-yr cycle phases, by $\mathrm{W}$ smoothed monthly values, as follows: the maximum phases were chosen so to encompass the whole structure with two maxima in $\mathrm{W}$ curve, the minimum phases were taken the whole intervals with $W<20$ and, the ascendant and descendant phases between the maximum and minimum ones. So, resulted the ascendant phases of Table 1 (column2), but we took in consideration the ascendant phases prolonged to the $\mathrm{W}$ maximum monthly values.

Table 1. Ascendant phases of the solar cycle nos. 20-23

\begin{tabular}{ccc}
\hline SC No. & Ascendant Phase & Ascendant Phase to W max. \\
\hline 20 & Nov.1965-Nov.1967 & Nov.1965-Nov.1968 \\
21 & Mar.1977-Feb.1979 & Mar.1977-Dec.1979 \\
22 & Mar.1987-Nov.1988 & Mar.1987-Jul.1989 \\
23 & Jun.1997-Aug.1999 & Jun.1997-Apr.2000 \\
\hline
\end{tabular}

The monthly values of $\mathrm{I}$ is presented by histograms in Figure 1 in comparison with the standard cyclic curve of sunspot relative number (smoothed values). The ascendant phases prolonged to the $\mathrm{W}$ maximum monthly value are marked by rectangles. 


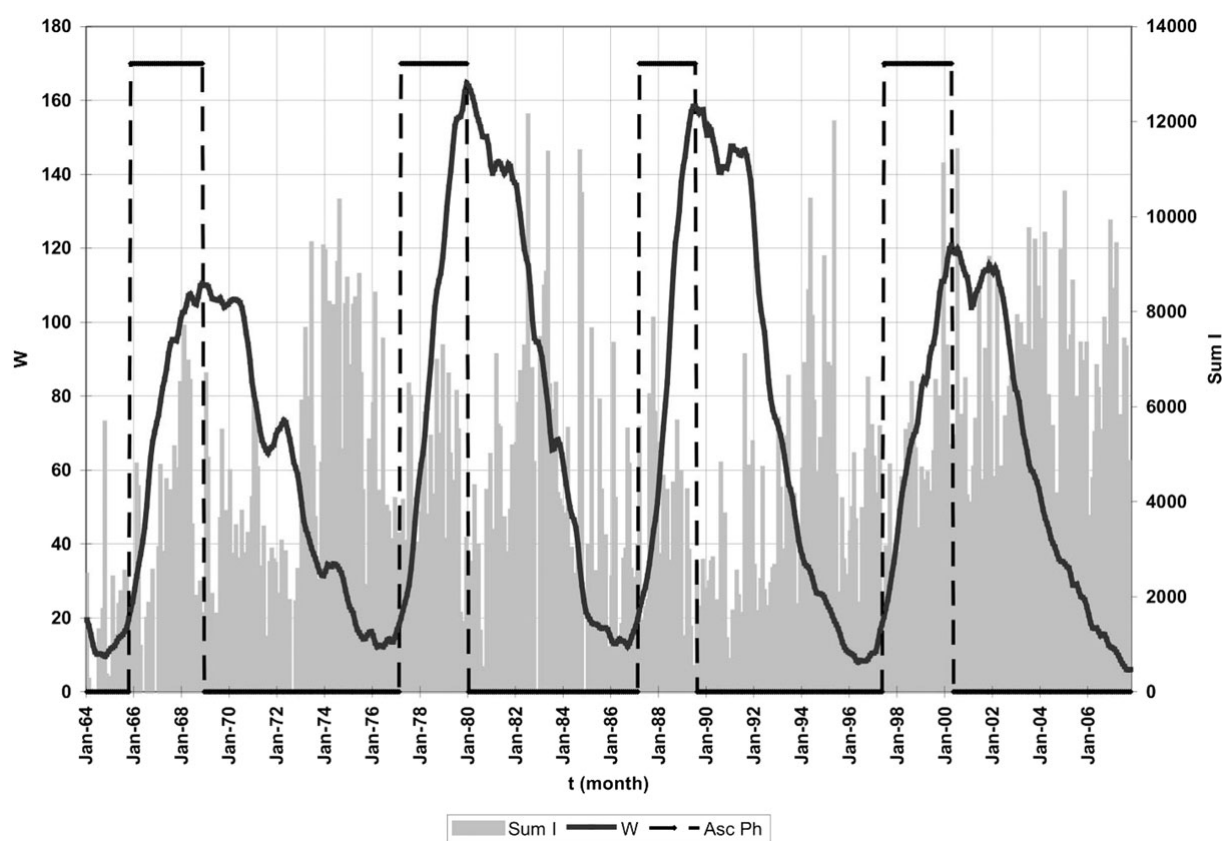

Figure 1. SC 20-23 ascending phases marked by rectangles (dotted line) on the W smoothed monthly plot and the HSS importance (I) histograms.

\section{Results and discussions}

The HSS intensity by monthly values shows less activity during ascendant phases in comparison with its values during the second part of the cycles (Fig. 1). The higher I values during ascendant phases of SCs 20 and 23 could be remarked even on the unsmoothed values (histogram) in Fig. 1.

We made a correlation analysis of the monthly values of Ap and I_HSS (smoothed values) applied on the whole interval (SCs 20-23) as well as on each ascendant phase of the cycles. The values of the correlation coefficient, $r$, are given in Table 2.

Table 2. Correlation coefficients between Ap and I_HSS series

\begin{tabular}{cc}
\hline Intervals & $\mathbf{r}$ \\
\hline Asc. phase of SC 20 & 0.39 \\
Asc. phase of SC 21 & 0.42 \\
Asc. phase of SC 22 & -0.14 \\
Asc. phase of SC 23 & 0.81 \\
SCs nos. 20 - 23 & 0.32 \\
\hline
\end{tabular}

The correlation coefficients between Ap and I_HSS are quite different in different cycles. We could draw attention on the highest coefficient in SC 23 that was one of the less intensive cycles in sunspot activity. The low correlation between Ap and I_HSS in SC 22 could be due to the gaps in data about the solar wind speed that did not allow a precise determination of HSSs. During ascendant phases of the SCs 20 and 21, the correlation coefficients between stream intensity and Ap are slightly higher than the one for the whole interval of SCs 20-23.

Generally, we could asses that the high-energy of the HSSs is not well enough reflected in the Ap index dynamics. The energy transfer from HSS into the terrestrial 
magnetosphere needs a magnetic fields reconnection that is more effective only when the $\mathrm{Bz}$ component of the interplanetary magnetic field has a southern orientation - the situation that we did not take into consideration.

It is well known that the data series could be analysed from two different standpoints: the time domain and the frequency domain. We applied a spectral analysis (Lomb-Scargle method) on the solar wind velocity series (hourly values) in order to put into evidence some peculiarities during the ascendant phases. This series was chosen in order to have longer series and considering that solar wind velocity gives a good evaluation of the wind

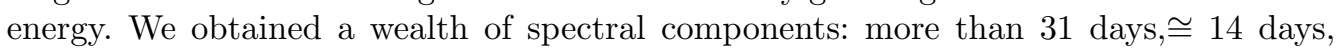
9 days - for even cycles and, 27-29 days, $\cong 13$ days, 9 days - for odds cycles. The Fast Fourier Transform method applied on the solar wind velocity series for SCs 20-23 interval revealed a main spectral component at 26.6 days and two components at 13.67 and 9 days (Parvutoiu et all, 2009). The same components were practically obtained for Aa index (25.7; 13.6 and, 8.9 days).

We note a main spectral component longer than the period of solar rotation (27 days) during the ascendant phases that could be imprint by the polar coronal holes, sources of HSS. Similar analysis will be applied on descendant phases of solar cycles.

\section{Acknowledgements}

The authors acknowledge the OMNI data, obtained from the GSFC/SPDF OMNI Web interface at http://omniweb.gsfc.nasa.gov. We also acknowledge the data from World Data Centre for Geomagnetism, Edinburgh (http://www.geomag.bgs.ac.uk/gifs/aaindex. html) and SIDC, Brussels (http://sidc.oma.be/sunspot-data/). One of the authors (GM) acknowledges the IAU travel grant to IAU General Assembly 2009, Rio de Janeiro.

\section{References}

Lindblad, B. A., Lundstedt, H., \& Larsson B. 1989, Sol. Phys 120, 145-152.

Maris, G. \& Maris, O. 2003, Rom. Astron. J. 13, 125-138.

Maris, O. \& Maris, G. 2005, Adv. Space Res. 35, 2129-2140.

Maris, O. \& Maris, G. 2008, unpublished yet; 1996-2007, at: http://www.spaceweather.eu/ in Cap. "Data Catalogs for $S W$ ".

Mavromichalaki, H. \& Vassilaki, A. 1998, Sol. Phys 183, 181-200.

Pirvutoiu, C., Vladoiu, D., Maris, G. 2009, in: SPACE PLASMA PHYSICS, edited by I. Zhelyaskov, (C)2009, American Institute of Physics, 978-0-7354-0656-8, pp. 141-150. 21292140 . 